\title{
Dynamic Evaluation Model of Urban Public Transport Service Level
}

\author{
Lang Liu \\ North China Electric Power University Baoding, Baoding 071000, China
}

2100639007@qq.com

\begin{abstract}
With today's urban public transport system's functions becoming increasingly perfect, how to make the dynamic evaluation of the existing traffic facilities and service based on the card consumption data of bus and the GPS data of taxi or other large data information, whose target is to improve the quality specially has gradually became a major concern to relevant government departments. In view of the given information and data, we make the following work to solve the problem of the topic proposed. We use the vehicle flow rate recognition algorithm and cross section calculation method after successfully importing the original data into MATLAB and making classification by screening in the database. According to the vehicle's GPS data, we finally work out a figure for bus and subway traffic statistics, and furthermore, forecast the citizens-traveling-figure in that city. On this basis, we explore a more intuitive citizens-traveling-histogram. At last, according to the information of time-determained passenger's number, the eventually mapped the entire network section of passenger flow rate information was created to reflect the city traffic flow changes of the different time and space. On the basis of above, added the GPS data and bus brush calorie of consumption data of residents, the matrix of OD was made to help deepen the comprehension.
\end{abstract}

Keywords: OD Matrix, Gauss filtering, The least square method

\section{Introduction}

In order to scientifically measure the running efficiency of public transportation, and provide accurate and reliable time information for public traffic network, it is necessary to develop a set of travel technology for public transport efficiency evaluation index and the corresponding key technology. As a whole, establishing an objective, system, scientific and reasonable evaluation index system is our mainly work because a comprehensive and objective evaluation of the urban public transportation development level can help reflect the city public transport state. In this question, we use the OD matrix, the OD key to and OD mainstream knowledge to transfer the GPS data and credit card consumer data information to the OD matrix. Once each OD matrix have got a certain time period of a certain specific location we multiply all the matrices, and then we get all the transfer information. 


\section{The OD Matrix Based on GPS Data}

\subsection{The concept of OD Matrix[1]}

OD matrix (OD traffic flow) is the traffic volume of traffic data, which is the basic requirement of the subway transportation network. It is important to carry out the traffic management and traffic management. The Metro OD matrix reflects the demand model of metro traffic in a given period of time. OD information based on the bus, subway, taxi GPS data can be used as follows:

- Real time and accurate grasp of the characteristics of the traffic flow and traffic characteristics of urban metro traffic, which provides the basis for the real-time deployment of the metro vehicles.

- Provide the basis for the deployment of public transport vehicles and public transport planning for the subway station.

-Decision support for the whole urban traffic planning scheme.

\subsection{The Establishment of OD Matrix}

Based on the $\mathrm{O}$ point, the traffic demand of the point to the point of $\mathrm{D}$ is calculated by the $\mathrm{O}$ point, and we call it the OD matrix regardless of the trip. Generally a platform for the OD in the region (city) of the $n$ matrix can be expressed as follows:

\begin{tabular}{|c|c|c|c|c|c|c|}
\hline $0 \quad$ D & 1 & 2 & ... & $j$ & $\ldots$ & $n$ \\
\hline 1 & $S_{11}$ & $S_{12}$ & $\ldots$ & $S_{1 j}$ & $\ldots$ & $S_{1 \mathrm{n}}$ \\
\hline 2 & $S_{21}$ & $S_{22}$ & ... & $S_{2 j}$ & $\ldots$ & $S_{2 \mathrm{n}}$ \\
\hline$\ldots$ & $\cdots$ &.- & $\ldots$ & $\ldots$ & $\ldots$ & $\ldots$ \\
\hline$i$ & $S_{i 1}$ & $S_{\text {i2 }}$ & $\ldots$ & $S_{i j}$ & $\ldots$ & $S_{\text {in }}$ \\
\hline 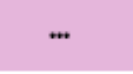 & $\cdots$ & $\cdots$ & $\ldots$ & $\cdots$ & $\cdots$ & $\ldots$ \\
\hline$n$ & $S_{n 1}$ & $S_{\mathrm{n} 2}$ & $\ldots$ & $S_{n j}$ & $\ldots$ & $S_{n n}$ \\
\hline
\end{tabular}

\subsection{Summary of OD Matrix}

For each passenger, there will be an OD Matrix which can reflect the change information of the passenger. For example, if we definite that if a passenger transfer for one time, we write number 1 at the intersection of two transfer points. So we 
collect all the passenger's OD information and then multiply all of the matrix, we can get the Shenzhen City's total passenger traffic information at a certain time.

\section{The urban traffic evaluation}

\section{1 the establishment of the evaluation model}

The first traffic conditions of forty sites were randomly selected from the existing data in the city of Shenzhen based on some five lines on the statistics and analysis in detail. It is shown to the reader by any of these lines, and then by using the Gauss filtering algorithm[2] we preprocess the data, and by using the least square method[3] we predict the future of Shenzhen city traffic bus arrival time.

Before modeling, we have the following several aspects of the whole system:

- passenger satisfaction

the effect of the pressure on the traffic

Othe situation of congestion

\subsection{Gauss filter}

Gauss filter is a linear smoothing filter, which is suitable for the elimination of Gauss noise, which is widely used in the process of image processing. In common, Gauss filtering is the process of weighted average of the whole matrix, and the value of each point is obtained by the weighted average of its own and other points in the neighborhood. The specific operation of the Gauss filter is to use a template (or a mask) to scan each point in the matrix, and the weighted average gray value of the neighborhood points is determined by the template to replace the value of the template center point. Gauss smoothing filter is very effective for suppressing the noise of normal distribution. This new vector diagram can also be used to further smooth the 3 x 3 template.We usually use the Gauss filter (that is, the template):

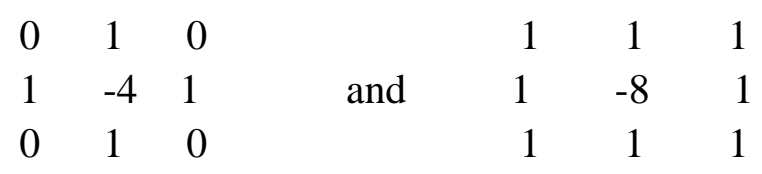

Then we use the Gauss filter on the OD matrix convolution operation, you can get the data matrix after the Gauss noise. 


\section{3 the least square method}

When a series of data is known, and the following form of this set of data is fitted with the least squares:

$$
y=A \sin (k x+b)+m
$$

Problem analysis and solving:

We know that there may be no solution for any real coefficient linear equations $A_{m \times n} x=b$, that is, any set of numbers $x_{1}, x_{2}, x_{3}, \ldots x_{n}$ may make the next formula set up:

$$
\|b-\mathrm{Ax}\|_{2=} \sum_{i=1}^{m}(\operatorname{ai} 1 \times 1+a i 2 \times 2+\cdots+\text { ainxn }-\mathrm{bi})_{2} \neq \mathbf{0}
$$

Now try to find the $x_{1}^{0}, x_{2}^{0}, x_{3}^{0}, \ldots x_{n}^{0}$ to make the constant $\|b-\mathrm{Ax}\|_{2}$ above smallest. And then use it as the approximate solution of a set of equations $A x=b$

\section{Model Summary}

\section{Advantages of the model:}

- $\quad$ by setting up the bus load rate, site congestion, congestion mileage, supply and demand, a multi factor problem is simplified, and the complexity of the solution is simplified.

- using passenger car satisfaction trans into the bus bypass, so as to turn the problem into a physical problem.

\section{Disadvantages of the model:}

because of the huge amount of data, it only analyzes the supply and demand of the bus and the effect of the subsidy on the supply and demand of the bus. For any location, if this method is feasible, if the model is used, and if the proposed scheme is reasonable, and has not made a clear conclusion. 


\section{References}

[1] Zhen-qi CHEN, Shu-qiang WANG, J. (2009). "Summary on Models and Methods of OD Matrix Estimation Based on Road Traffic Flow.” LOGISTICS TECHNOLOGY, 28(8), 89-91.

[2] T. Tang, H.H. Hui, "Gaussian Curve filting solution based on Matlab", Computer \&Digital Engineering, vol. 41, no. 8, pp. 1262-1263, 2013.

[3] Y. Yongsheng, W. Haiyan, W. Xuan, "A novel least-square method of source localization based on acoustic energy measurements for uwsn", Signal Processing Communications and Computing (ICSPCC) 2011 IEEE International Conference, pp. 1-5, Sept 2011. 